\title{
Targeted disruption of MCPIP1/Zc3h12a results in fatal inflammatory disease
}

\author{
Ruidong Miao ${ }^{1,2,7}$, Shengping Huang ${ }^{1,7}$, Zhou Zhou ${ }^{3,7}$, Tim Quinn ${ }^{1}$, Benjamin Van Treeck ${ }^{1}$, Tehreem Nayyar ${ }^{1}$, \\ Daniel Dim ${ }^{4}$, Zhisheng Jiang ${ }^{5}$, Christopher J Papasian', Y Eugene Chen ${ }^{3}$, Gang Liu ${ }^{6}$ and Mingui Fu ${ }^{1}$ \\ Previous studies using MCP-induced protein 1 (MCPIP1)/Zc3h12a-deficient mice suggest that MCPIP1 is an important \\ regulator of inflammation and immune homeostasis. However, the characterization of the immunological phenotype of MCPIP1- \\ deficient mice has not been detailed. In this study, we performed evaluation through histological, flow cytometric, enzyme- \\ linked immunosorbent assay and real-time PCR analysis and found that targeted disruption of MCPIP1 gene leads to fatal, \\ highly aggressive and widespread immune-related lesions. In addition to previously observed growth retardation, splenomegaly, \\ lymphoadenopathy, severe anemia and premature death, MCPIP1-deficient mice showed disorganization of lymphoid organs, \\ including spleen, lymph nodes and thymus, and massive infiltration of lymphocytes, macrophages and neutrophils into many \\ other non-lymphoid organs, primarily in lungs and liver. Flow cytometric analysis found significant increase in activated and \\ differentiated T cells in peripheral blood and spleen of MCPIP1-deficient mice. Moreover, heightened production of \\ inflammatory cytokines from activated macrophages and T cells were observed in MCPIP1-deficient mice. Interestingly, \\ treatment of MCPIP1-deficient mice with antibiotics resulted in significant improvement of life span and a decrease in \\ inflammatory syndrome. Taken together, these results suggest a prominent role for MCPIP1 in the control of inflammation and \\ immune homeostasis.
}

Immunology and Cell Biology (2013) 91, 368-376; doi:10.1038/icb.2013.11; published online 9 April 2013

Keywords: MCPIP1; autoimmune disease; inflammation; cytokine; lymphocyte

As the immune system is continuously bombarded with foreign antigens, complex regulatory mechanisms interact to maintain immune homeostasis. Specifically, the inflammatory and immune responses must be sufficient to eliminate invading antigens, but the inflammatory response should not be sustained after the threat has been eliminated and, ideally, the invader should be eliminated without substantial collateral damage to the surrounding tissues. ${ }^{1,2}$ The molecular mechanisms contributing to the maintenance of immune homeostasis are not fully understood. We and others recently identified a novel member of $\mathrm{CCCH}$-zinc finger protein family, designated as MCP-induced protein 1 (MCPIP1) (also known as $\mathrm{Zc} 3 \mathrm{~h} 12 \mathrm{a}$ ), which is $65.8 \mathrm{kDa}$ protein encoded by the immediateearly response gene, $Z c 3 h 12 a .^{3-7}$ This gene has been mapped to chromosome 4 in mouse, and the equivalent gene in human, $\mathrm{ZC} 3 \mathrm{H} 12 \mathrm{~A}$, has been mapped to chromosome 1p34.3. In adult mouse, MCPIP1 mRNA is highly expressed in the lung, intestine, colon, lymph node, spleen and thymus. MCPIP1 mRNA is expressed at lower levels in the stomach, bladder, adipose tissue and the aorta, and is essentially absent in the brain, kidney, liver, heart and skeletal muscle. ${ }^{8}$ Several members of the MCPIP1 family, including MCPIP1/ Zc3h12a, Zc3h12b, Zc3h12c and Zc3h12d, were previously reported to contribute to negative regulation of lipopolysaccharide-induced macrophage activation. ${ }^{4}$ These initial findings were further confirmed by observations in MCPIP1-deficient mice. ${ }^{8,9}$ MCPIP1-deficient mice develop normally in utero. However, with age, they begin to suffer from spontaneous inflammatory diseases characterized by multiorgan inflammation, splenomegaly, heightened inflammatory cytokine production and premature death. ${ }^{89}$ MCPIP1-deficient cells are hyper-responsive to Toll-like receptor signaling, and MCPIP1deficient mice are hypersensitive to septic shock. ${ }^{10}$ Furthermore, we and others have recently shown that MCPIP1 is a multifunctional protein that actively participates in several distinct signaling pathways. For example, MCPIP1 downregulates lipopolysaccharide-induced inflammatory responses by acting as an RNase; ${ }^{9,11}$ MCPIP1 inhibits $\mathrm{C}$-Jun $\mathrm{N}$-terminal kinase (JNK) and nuclear factor- $\mathrm{KB}$ signaling by interfering with the ubiquitination of upstream signaling molecules,

${ }^{1}$ Department of Basic Medical Science, School of Medicine, University of Missouri-Kansas City, Kansas City, MO, USA; ${ }^{2}$ School of Life Sciences, Lanzhou University, Lanzhou, PR China; ${ }^{3}$ Cardiovascular Center, Department of Internal Medicine, University of Michigan Medical Center, Ann Arbor, MI, USA; ${ }^{4}$ Department of Pathology, University of Missouri-Kansas City, Kansas City, MO, USA; ${ }^{5}$ Institute of Cardiovascular Disease, School of Medicine, University of South China, Hengyang City, Hunan Province, PR China and ${ }^{6}$ Division of Pulmonary, Allergy and Critical Care Medicine, University of Alabama at Birmingham, Birmingham, AL, USA

${ }^{7}$ These authors contributed equally to this work.

Correspondence: Dr M Fu, Department of Basic Medical Science, School of Medicine, University of Missouri Kansas City, 2411 Holmes Street, Kansas City, MO 64108, USA E-mail: fum@umkc.edu

Received 12 November 2012; revised 2 March 2013; accepted 5 March 2013; published online 9 April 2013 
such as TNF receptor associate factors. ${ }^{8}$ These results collectively suggest that MCPIP1 is a novel antiinflammatory protein that negatively regulates both innate and adaptive immunity, and that its selective expression in lymphoid and inflamed tissues suppresses hyper-responsiveness, thereby contributing to the maintenance of immune homeostasis.

The goal of the current study was to further characterize the phenotypes of MCPIP1-deficient mice through histological, flow cytometric, enzyme-linked immunosorbent assay and real-time PCR analysis. We found that targeted disruption of MCPIP1 gene resulted in fatal disseminated inflammation, which was characterized by disrupted architecture of primary and secondary lymphoid organs, leukocytic infiltration of lungs and liver, activation of $\mathrm{T}$ cells and $\mathrm{B}$ cells and enhanced levels of both macrophage and $\mathrm{T}$ cell-derived cytokines. These results demonstrated a prominent role for MCPIP1 in control of inflammation and immune homeostasis.

\section{RESULTS}

Primary and secondary lymphatic tissues of MCPIP1-deficient mice exhibit architectural disorganization

Despite the striking phenotypic changes reported for MCPIP1 $1^{-/-}$ mice in our previous study, ${ }^{8}$ the detailed pathogenesis of these alterations was not fully analyzed. Consquently, in the current study, we further characterized the changes occurring in the major organs and tissues, including the thymus, spleen, lymph nodes, lungs, liver, heart, aorta, kidney, skin and intestine and so on, from MCPIP1-deficient mice by histological, flow cytometric and realtime PCR analysis. The most strinking morphological changes were observed in the spleen, lymph nodes and thymus of MCPIP1deficient mice. As shown in Figure 1a, the architecture of the spleen, lymph nodes and thymus from both male and female MCPIP1deficient mice at 8 weeks of age appeared disorganized. In the spleen, the white pulp appeared underdeveloped, whereas the red pulp had marked expansion, probably a result of extramedullary hematopoiesis. Such pathology disrupted normal splenic architecture. In lymph nodes of MCPIP1-deficient mice, normal follicular architecture of the cortex was missing, which was probably caused by underdevelopment. The paracortical areas were also disorganized. In the thymus, the cortex was essentially absent and the medulla was significantly expanded. To investigate the development of this architectural disruption with age, we futher observed the phenotype of MCPIP1deficient mice at the ages of 2 days, 3 weeks and 6 weeks. As shown in Figures $1 \mathrm{~b}-\mathrm{d}$, the gross size and histological morphology of spleens from MCPIP $1^{-l-}$ mice at the age of 2 days were essentially normal compared with those from wild-type littermates. By 3 weeks of age, spleens from MCPIP $1^{-1}$ - mice were obviously larger than those from MCPIP1 $1+1+$ littermates, and these differences became even more pronounced by 6 weeks of age. These results suggest that the architectural abnormalities observed in primary and secondary lymphatic tissues of MCPIP1-deficient mice progressed with age.

\section{MCPIP1-deficient mice exhibit multiorgan inflammation}

The gross architecture of the non-lymphoid organs from MCPIP1deficient mice was essentially normal compared with that from wildtype littermates (at the age of 8 weeks). Microscopically, however, multifocal and mixed inflammatory cell infiltration was observed in many of these organs, with the most severe changes occurring in the lungs and liver from MCPIP1-deficient mice (Figure 2 and Supplementary Figure S1). At 3 weeks of age, leukocyte infiltration into the interstitial spaces of the lungs was observed in MCPIP1 $1^{-1-}$ mice, but not in MCPIP $1^{+/+}$mice (Figure $2 \mathrm{a}$ ). By 8 weeks of age, massive infiltration of leukocytes into the interstitial spaces of the lungs, marked by thickening of the alveolar septa and pulmonary edema were observed in MCPIP1 $1^{-/-}$mice, but not in MCPIP1 $1^{+/+}$mice. Large amounts of mucus also filled the terminal bronchioles and alveolar spaces of MCPIP1 $1^{-/}$mice by 8 weeks. In the liver, inflammatory cell infiltration in the portal triad area, with infiltrates most concentrated near the bile ducts, was observed in MCPIP1 ${ }^{-1-}$ mice, but not in MCPIP1 ${ }^{+1+}$ mice (Figure 2b). Mild-to-moderate inflammatory cell infiltration was also observed in the intestines, and kidneys from MCPIP1 ${ }^{-1-}$ mice. It was also noted that transmural thickening of the intestine also appeared in MCPIP1 $1^{-/-}$mice. In the kidneys of $\mathrm{MCPIP}^{-1-}$ mice, the glomeruli were larger with a skewed matrix to nuclei ratio (Supplementary Figure S1). There was no microscopic evidence of bacterial invasion into these tissues. No significant inflammation was observed in other tissues of $\mathrm{MCPIP}^{-1-}$ mice, such as heart, aorta and skin, despite there was some minor inflammatory cell infiltration into adipose tissue surrounding the aorta (Supplementary Figure S1). Immunohistochemical staining demonstrated that most of the inflammatory cells in the lungs were B lymphocytes and macrophages, whereas in the liver, predominately neutrophils and macrophages (Figure 2c).

\section{MCPIP1-deficient mice exhibit increased immunoglobulin accumulation in multiple tissues}

Previous report showed that MCPIP1/Zc3h12a null mice developed hyperimmunoglobulinemia of all immunoglobulin (Ig) isotypes. ${ }^{9}$ Antinuclear antibodies and anti-double-stranded-DNA antibodies were also detected in MCPIP1/Zc3h12a null mice. ${ }^{9}$ In the current study, immunofluorescent staining revealed increased accumulation of IgM and IgG in multiple organs from MCPIP $1^{-1-}$ mice, including spleen, skin, lungs and aorta, but not in those from MCPIP $1^{+1+}$ mice (Figure 3). Interestingly, the accumulation of immune complex was not obvious in the kindey from MCPIP1 ${ }^{-1-}$ mice (Figure 3 ). Nevertheless, these results demonstrated that MCPIP1-deficient mice spontanously developed an autoimmune response.

\section{Immune cell abnormalities in MCPIP1-deficient spleens}

To further characterize the cellular components of the systemic inflammation in MCPIP1-deficient mice, we performed flow cytometry analysis of cell populations in the spleens of $\mathrm{MCPIP}^{-1-}$ and MCPIP $1^{+}++$mice. First, we analyzed nucleated spleen cells to determine the percentage of $\mathrm{B}$ cells, $\mathrm{T}$ cells, neutrophils and monocytes, and found that the percentage of B cells in the spleen was dramatically decreased, the percentage of neutrophils was markedly increased, and the percentage of $\mathrm{T}$ cells was moderately decreased in MCPIP1 $1^{-/}$mice; monocytes remained essentially unchanged (Figure 4a). Interestingly, although B cells represented a relatively small percentage of nucleated cells in the spleens of MCPIP $1^{-1-}$ mice $(\sim 7 \%)$, a surprisingly high proportion of these cells had differentiated into plasma cells $(\sim 25 \%)$ (Figure $4 \mathrm{a})$. To further explore lymphocyte activation within the spleen, we studied various T-cell populations. Specifically, we compared the levels of activation of $\mathrm{CD}^{+}{ }^{+}$and $\mathrm{CD} 8{ }^{+}$cells from the spleens of MCPIP1 ${ }^{-1-}$ and MCPIP $1^{+I+}$ mice using the specific markers for activated T cells (CD62L), Th1 (interferon, IFN- $\gamma$ ), and Th2 (interleukin-4, IL-4). As shown in Figures $4 \mathrm{~b}$ and $\mathrm{c}, \mathrm{CD} 62 \mathrm{~L}^{\text {low }}$ was significantly upregulated in both $\mathrm{CD}^{+}$and $\mathrm{CD} 8{ }^{+}$cells from MCPIP1 ${ }^{-1-}$ mice compared with those from MCPIP1 $1^{+/+}$mice, suggesting that $\mathrm{T}$ cells were automaticlly activated in MCPIP1 $1^{-1-}$ mice. Moreover, INF $\gamma$ was also significantly upregulated in both $\mathrm{CD}^{+}$and $\mathrm{CD}^{+}$cells from $\mathrm{MCPIP}^{-l-}$ mice and IL-4 was significantly elevated in CD4 ${ }^{+}$cells, 


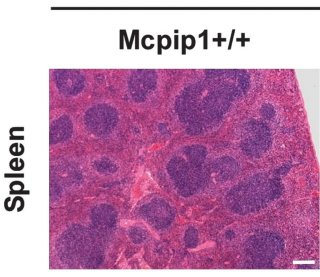

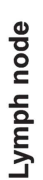

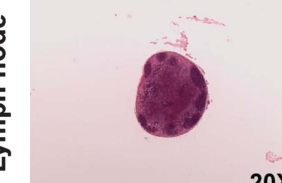

20X
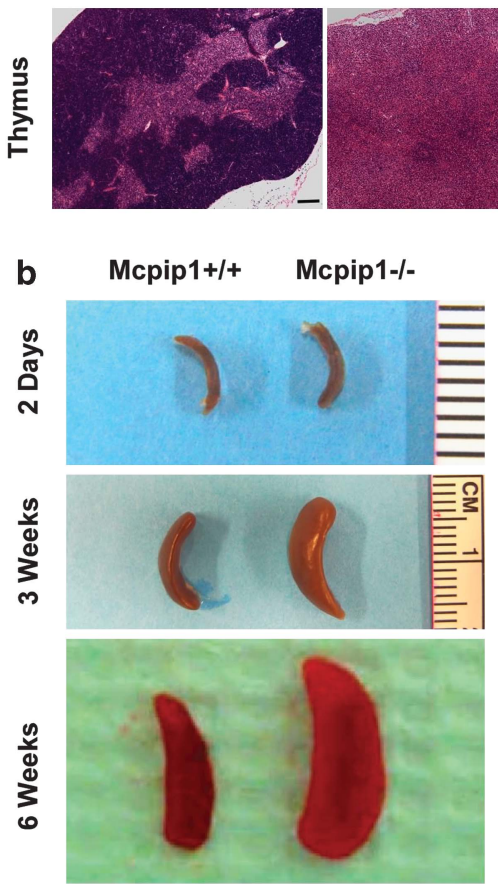

d

2 Days

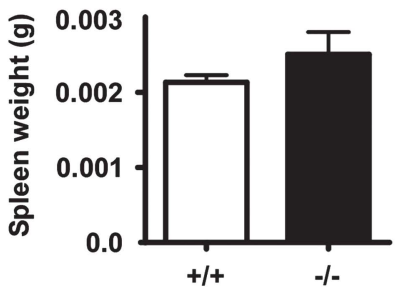

C

20x

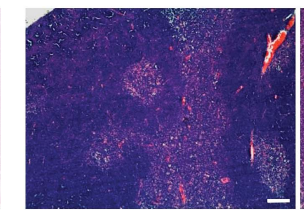

Mcpip1+/+
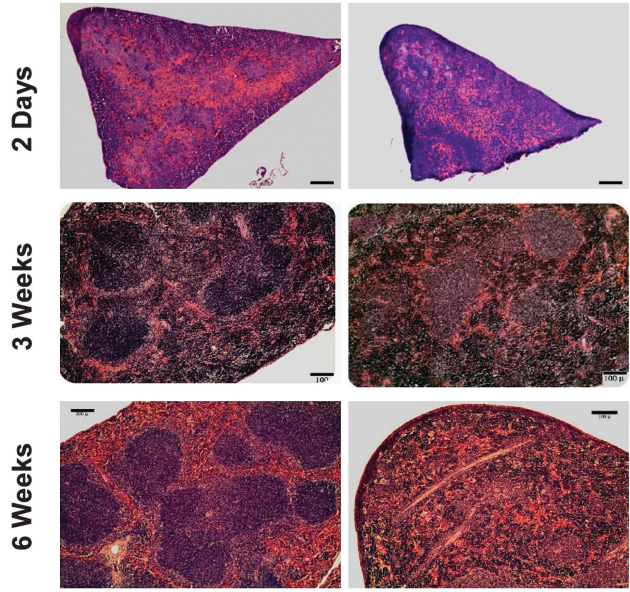

3 Weeks

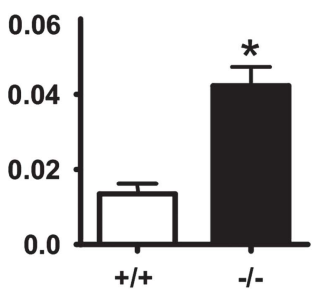

6 Weeks

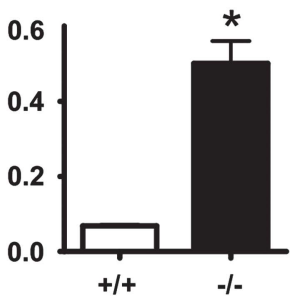

Figure 1 Disorganization of lympoid organs from MCPIP1 ${ }^{-/}$- mice. (a) Hematoxylin and eosin (H\&E) staining of formalin-fixed spleen, lymph node and thymus sections from both male and female MCPIP1 ${ }^{-/-}$mice and wild-type littermates (MCPIP1 ${ }^{+/+}$) at the age of $8-10$ weeks. Magnitude is $\times 100$ except indicated. Bar $=100 \mu \mathrm{m}$. (b) Gross morphology of the spleen from MCPIP1 $1^{+/+}$and MCPIP1 ${ }^{-1-}$ mice at the indicated age. (c) H\&E staining of the spleen from MCPIP1 $1^{+/+}$and MCPIP1 ${ }^{-l-}$ mice at the indicated age. Bar $=100 \mu \mathrm{m}$. (d) The spleen weight from MCPIP1 $1^{+/+}$and MCPIP1 ${ }^{-l-}$ mice at the indicated age. $N=3 .{ }^{*} P<0.05$ vs wild-type group.

but not in $\mathrm{CD}^{+}$cells of $\mathrm{MCPIP} 1^{-} /^{-}$mice (Figure 4c). These findings support that lymphocyte activation and differentiation were profoundly enhanced in the spleens of MCPIP1 $1^{-1-}$ compared with that in MCPIP $1^{+1+}$ mice. In addition, the percentage of both $\mathrm{CD} 4{ }^{+}$ and $\mathrm{CD}^{+}$cells expressing IL-17 was significantly increased in MCPIP $1^{-l-}$ compared with MCPIP $1^{+/+}$mice (Figure 4c). In view of the profound proinflammatory effects of IL-17, it is probable that enhanced expression of IL-17 in MCPIP1 ${ }^{-1-}$ mice contributed to acute inflammatory changes observed within the spleens from these mice. Somewhat surprisingly, Foxp3-expressing cells were also more prominent in the spleens from MCPIP $1^{-1}$ - mice, particularly among $\mathrm{CD}^{+}$cells (Figures $4 \mathrm{~b}$ and c). Consequently, the profound inflammatory response in the spleens of $\mathrm{MCPIP}^{-1-}$ mice could not be attributed to deficient numbers of $\mathrm{T}_{\text {reg }}$ cells. 

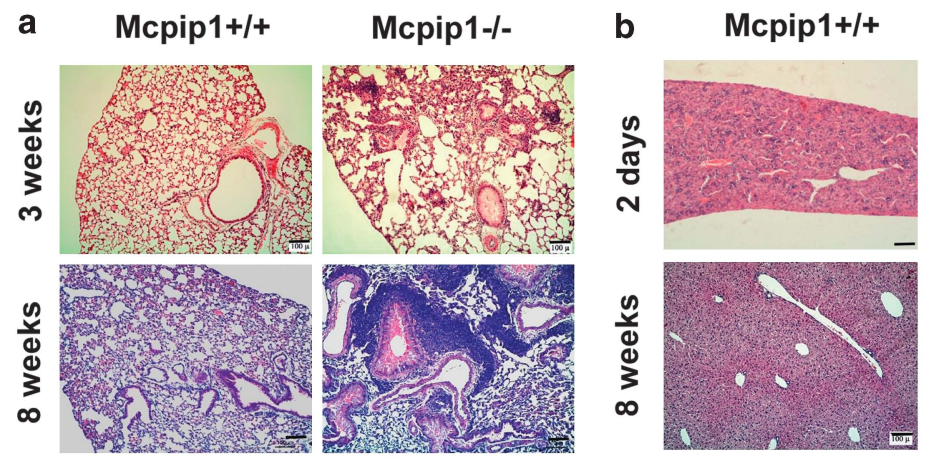

Mcpip1-/-

C

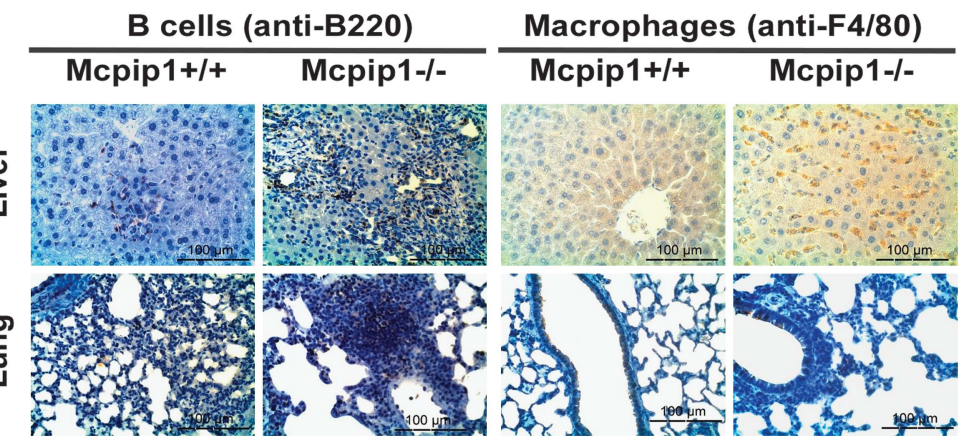

Figure 2 Development of multiorgan inflammation in MCPIP1 $1^{-1-}$ mice. (a) H\&E staining of formalin-fixed lung sections from MCPIP1 ${ }^{-1-}$ mice and wildtype littermates at the indicated age. (b) H\&E staining of formalin-fixed liver sections from MCPIP1 ${ }^{-l-}$ mice and wild-type littermates at the indicated age. (c) Immunohistochemical staining of formalin-fixed liver and lung sections from MCPIP1 null mice and wild-type littermates at the age of 8 weeks with B-cell marker (anti-B220) and macrophage marker (anti-F4/80).

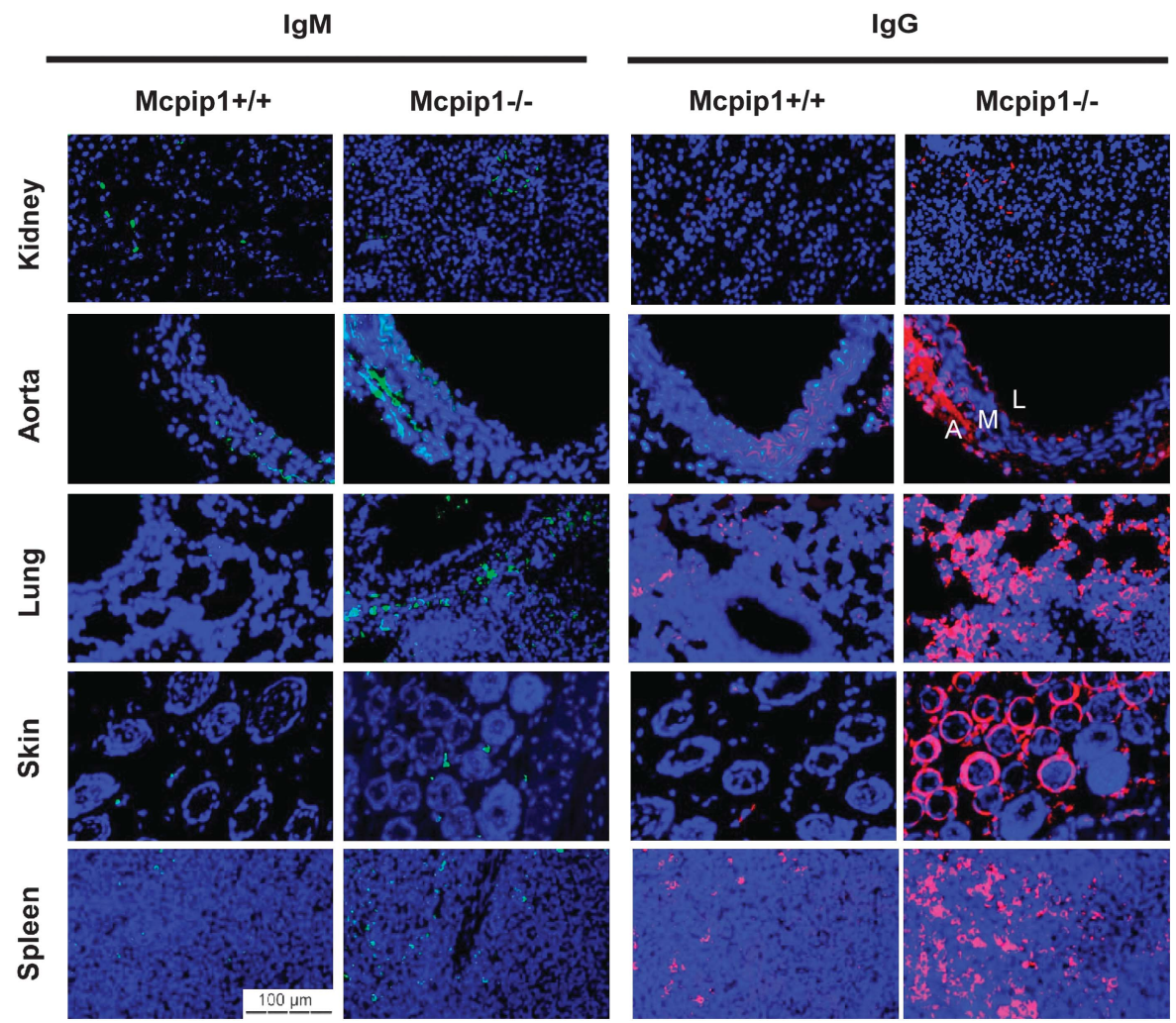

Figure 3 Ig accumulation in MCPIP1 ${ }^{-1-}$ mice. Immunofluoresence staining of formalin-fixed kidney, aorta, lung, skin and spleen sections from MCPIP1 ${ }^{-1-}$ mice and wild-type littermates at the age of 8 weeks with anti-lgM and anti-lgG. The layers of aorta have been marked as 'A' (adventitial), 'M' (media layer) and 'L' (lumen). 

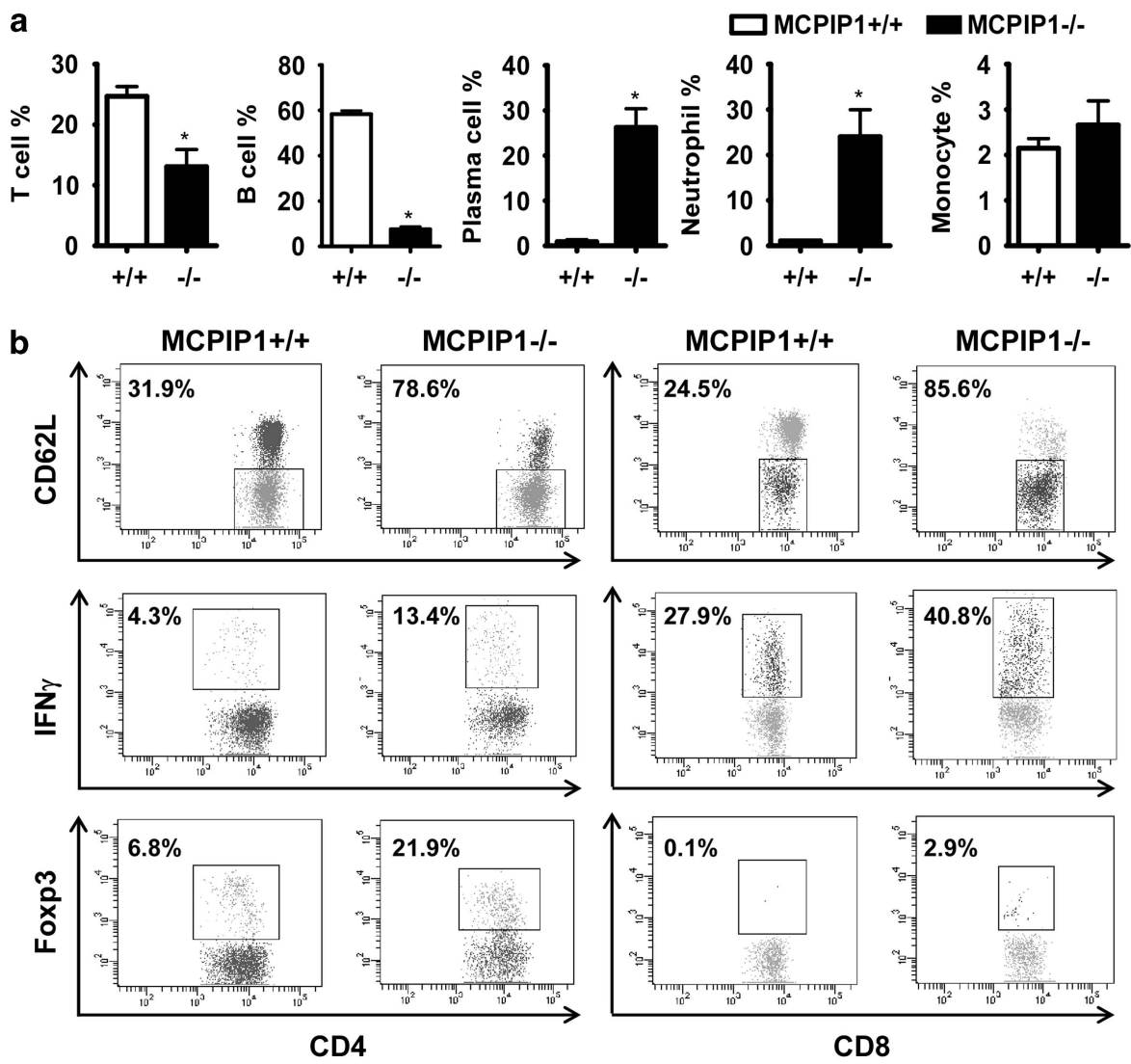

C
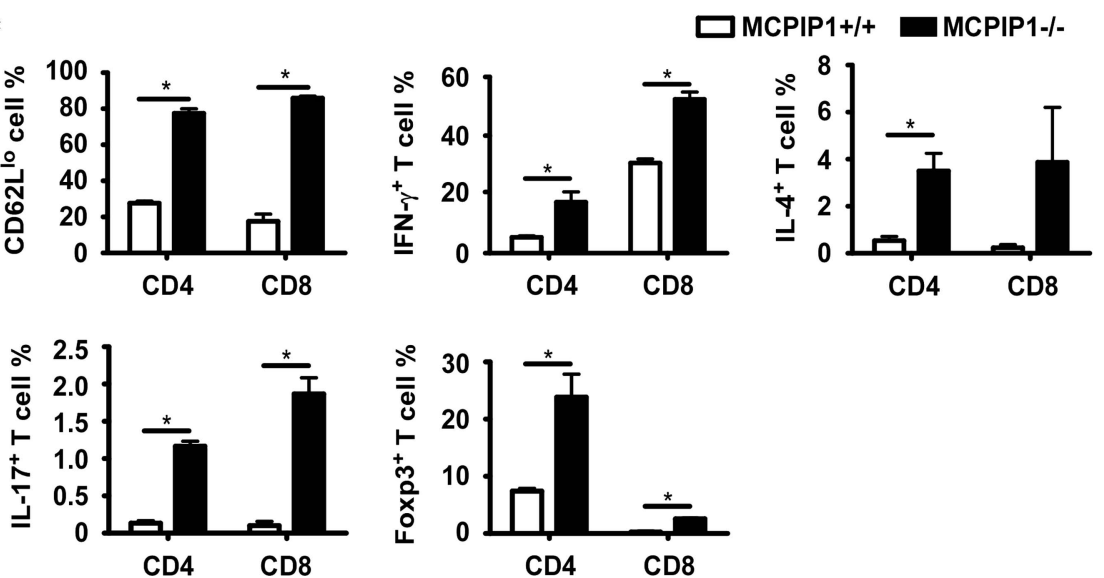

Figure 4 Immune-cell abnormalities in MCPIP1-deficient spleens. Single-cell suspensions of spleens from 6-week-old wild-type and MCPIP1 ${ }^{-/-}$mice were counted and stained for flow cytometric analysis. (a) The percentage of total T cells, B cells, neutrophils and monocytes in total nucleared cells, and the percentage of $\mathrm{B}$ cells that had differentiated to plasma cells were reported as mean \pm s.d., $n=3$. ${ }^{*} P<0.01$ vs wild-type mice. (b) Flow cytometry plots for $\mathrm{CD} 62 \mathrm{~L}$, IFN $\gamma$, and Foxp3 in $\mathrm{CD}^{+}{ }^{+}$and $\mathrm{CD}^{+}$cells. (c) The percentage of subtypes of $\mathrm{T}$ cells in both $\mathrm{CD}^{+}{ }^{+}$and $\mathrm{CD} 8{ }^{+} \mathrm{T}^{-}$-cell populations were reported as mean \pm s.d., $n=3 .{ }^{*} P<0.01$ vs wild-type mice.

MCPIP1-deficient mice exhibit increased protein and mRNA levels for markers of innate and adaptive immunity

To further delineate the molecular mechanisms underlying the fatal inflammation in MCPIP $1^{-1-}$ mice, we examined protein level of 23 proinflammatory cytokines, chemokines and other markers of inflammation in serum of 2-month-old mice (not all data are shown). As shown in Figure 5a, the proinflammatory cytokines from both innate cells (such as IL-1, IL-6, monocyte chemotactic protein-1 and TNF $\alpha$ ) and adaptive immune cells (such as IL-2, IL-3, IL-4, IL-5,
IL-12p40, IL-13, IL-17 and IFN $\gamma$ ) were all increased in the serum from MCPIP $1^{-/}$mice compared with that from MCPIP $1^{+/+}$mice. Moreover, the mRNA levels for MD2, Toll-like receptor4, CD14 and IL-6 were significantly increased in the spleens from MCPIP1 $1^{-1-}$ compared with MCPIP1 $1^{+/+}$mice (Figure 5b). No significant differences were observed in mRNA levels for TNF $\alpha$, IL-1 $\beta$, monocyte chemotactic protein-1, and inducible nitric oxide synthase in the spleens from both genotypes, suggesting that other sources may contribute the expression levels of these inflammatory cytokines. 

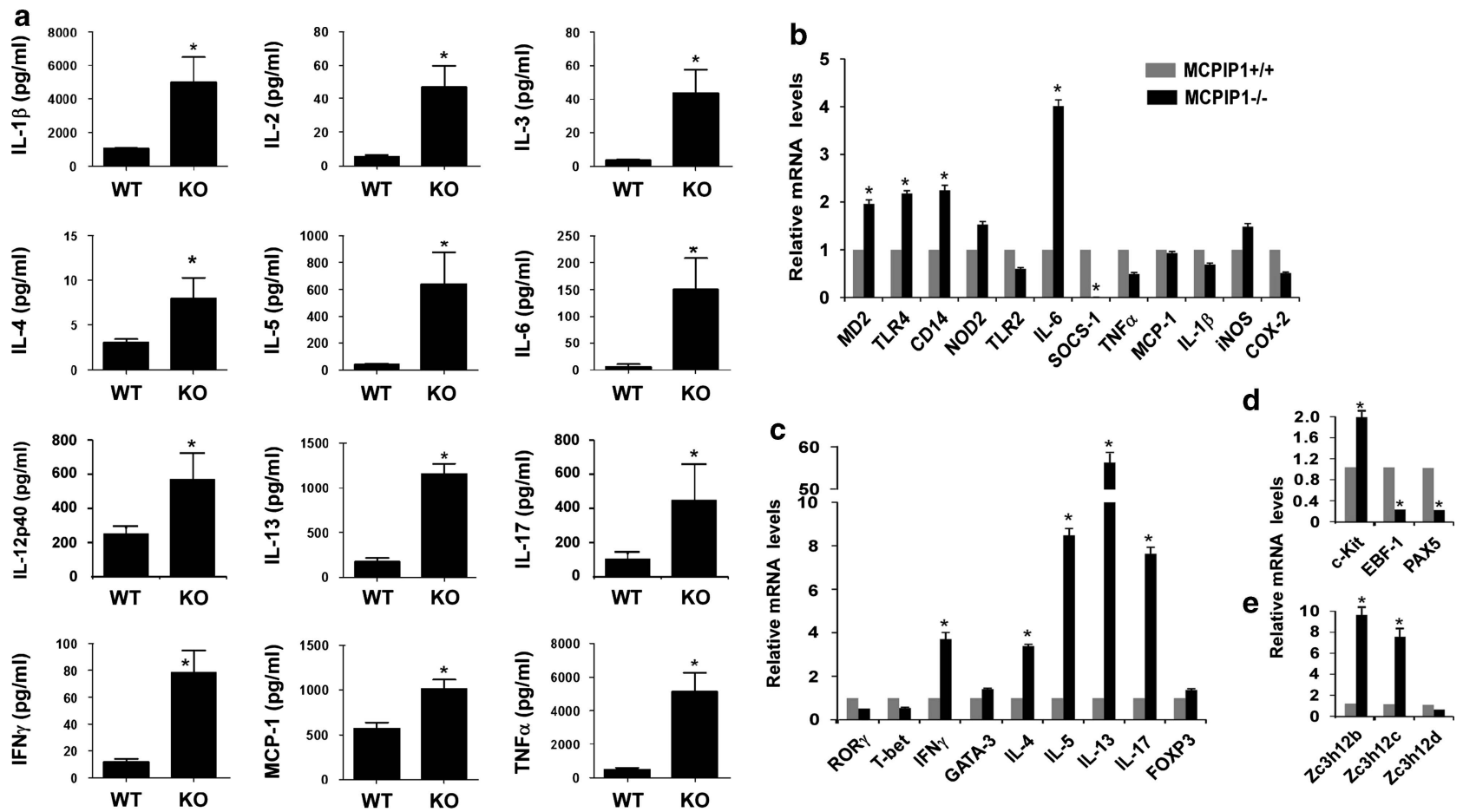

Figure 5 Expression of proinflammatory cytokines, chemokines and other markers of inflammation from MCPIP1 $1^{-l-}$ and MCPIP1 $1^{+l+}$ mice. (a) Enzymelinked immuno sorbent assay analysis of serum levels of proinflammatory cytokines, chemokines and other markers of inflammation of MCPIP1-deficient mice (KO) and their wild-type littermates (WT) at the age of 6-8 weeks ( $n=3$ per genotype). Data are presented as mean \pm s.d., $n=3$. ${ }^{*} P<0.01$ vs wildtype mice. (b-d) Quantitative PCR (QPCR) analysis of mRNA levels of proinflammatory cytokines, chemokines and other markers of inflammation in spleens of MCPIP1-deficient mice and their wild-type littermates at the age of 6-8 weeks ( $n=3$ per genotype). Data are presented as mean \pm s.d., $n=3$. ${ }^{*} P<0.01$ vs wild-type mice. (e) QPCR analysis of the mRNA levels of Zc3h12b, Zc3h12c and Zc3h12d in the spleens of MCPIP1-deficient mice and their wild-type littermates at the age of $6-8$ weeks ( $n=3$ per genotype).

Consistent with the results above, the mRNA levels for many T-cell cytokines, including IFN- $\gamma$, IL-4, IL-5, IL-13 and IL-17 were markedly elevated in $\mathrm{MCPIP}^{-/-}$compared with $\mathrm{MCPIP} 1^{+/+}$mice (Figure 5c). However, the expression levels of T-bet, GATA3 and ROR $\gamma$ were not changed, suggesting that MCPIP1 may directly regulate the mRNA degradation of IFN- $\gamma$, IL-4 and IL-17, but not their transcription. Among the B-cell markers, the expression marker of B-cells progenitors, c-Kit, was upregulated in MCPIP1 $1^{-/}$spleens; however, the expression of transcription factors for B-cells development, early B-cell factor 1 and Pax5, was downregulated in MCPIP1deficient mice (Figure 5d). Interestingly, the expression of both Zc3h12b and Zc3h12c, but not Zc3h12d were compensatory increased in the spleen from MCPIP1-l- mice (Figure 5e). Taken together, these results further demonstrated that the fatal inflammatory syndrome in MCPIP1 ${ }^{-l-}$ mice was associated with an abnormal response of both innate and adaptive immune cells.

\section{Antibiotic treatment improves the life span and inflammatory syndrome of MCPIP1-deficient mice}

The indigenous microbiota of mucosal surfaces, particularly the intestinal microflora, accounts for a substantial proportion of the stimulus to our innate and adaptive immune systems. ${ }^{12,13}$ To test whether the indigenous microbiota contributed to the development of inflammatory phenotypes of MCPIP1-deficient mice, we treated MCPIP1-deficient and wild-type mice from birth to 10 weeks with a combination of oral antibiotics that would greatly deplete the microflora of the intestine and other mucosal surfaces. As shown in Figure $6 \mathrm{a}$, the survival rate of MCPIP1-deficient mice was significantly improved at 10 weeks by antibiotic treatment; $80 \%$ of untreated MCPIP $1^{-1-}$ died, whereas only $33 \%$ of antibiotic $\mathrm{MCPIP}^{-1-}$ died by 10 weeks of age. Spleens from antibiotic-treated MCPIP1-deficient mice were much smaller than those from untreated MCPIP1-deficient mice (Figure 6b). Furthermore, the mRNA expression levels encoding inflammatory cytokines, such as IFN- $\gamma$, IL-13 and IL-17, but not IL-6, were significantly decreased in the spleens from antibiotic-treated MCPIP1-deficient mice compared with untreated mice (Figure 6c).

\section{DISCUSSION}

The key findings of the current study are that: (1) primary and secondary lymphoid organs of MCPIP1-deficient mice had signficant architectural abnormalities, and these abnormalities progressed with age; (2) MCPIP1-deficient mice spontanously developed profound, age-dependent, systemic inflammatory responses that involved both innate and adaptive immunity, as evidenced by marked infiltration of neutrophils, lymphocytes and macrophages into the lungs and liver, and by increased deposition of $\operatorname{IgG}$ and IgM in the spleen, skin and lungs; (3) overall cellular composition of the spleen was markedly affected by MCPIP1 deficiency, as evidenced by greatly decrease in the numbers of B cells, marked increase in the numbers of neutrophils, slight decreases in $\mathrm{T}$ cells, and an increase in the proportion of the B-cell lineage that had differentiated into plasma cells; (4) abnormal T-cell populations in the spleen of MCPIP1deficient mice that demonstrated enhanced immune activation; and (5) protein and mRNA levels for numerous markers of innate and adaptive immunity were elevated in the sera and spleens from 
a
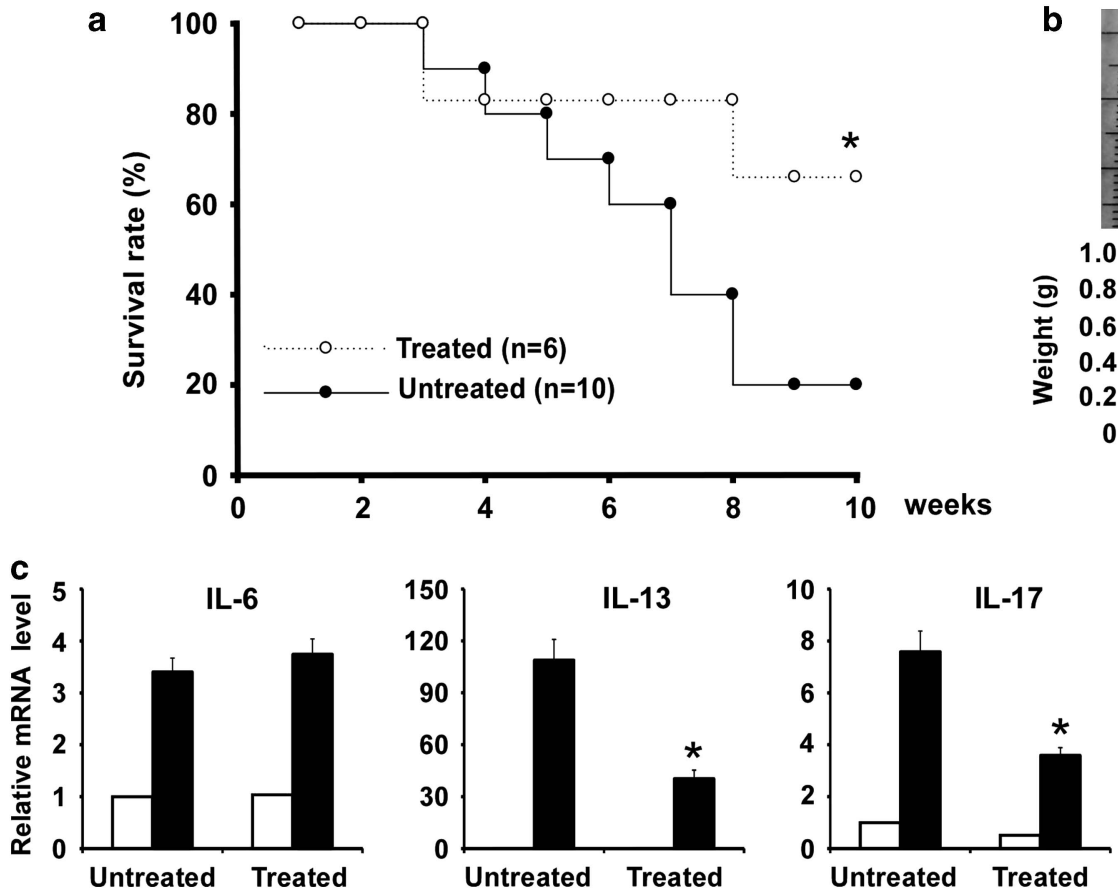

b
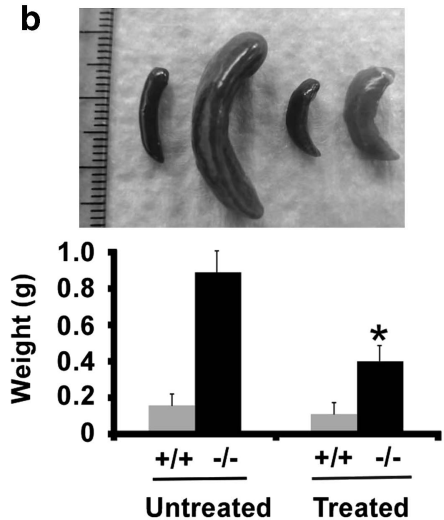

10 weeks

Untreated Treated

Figure 6 Antibiotic treatment improved the life span and inflammatory syndrome of MCPIP1 $1^{-1-}$ mice. (a) MCPIP1-deficient mice were treated orally with the drinking water containing ampicillin $\left(1 \mathrm{gl}^{-1}\right)$, neomycin $\left(1 \mathrm{gl} \mathrm{I}^{-1}\right)$, vancomycin $\left(\left.0.5 \mathrm{~g}\right|^{-1}\right)$ and metronidazole $\left(\left.1 \mathrm{~g}\right|^{-1}\right)(n=6)$ or normal drinking water $(n=10)$ from birth to 10 weeks of age. The survival rates from both groups were recorded and plotted. ${ }^{*} P<0.05$. (b) Representive gross morphology and weight of spleens from antibiotic treated and untreated MCPIP1 ${ }^{+/+}$and MCPIP1 ${ }^{-1-}$ mice. ${ }^{*} P<0.05, n=3$. (c) QPCR analysis of mRNA levels of proinflammatory cytokines in the spleens from antibiotic treated and untreated MCPIP1 ${ }^{+/+}$and MCPIP1 ${ }^{-/-}$mice. Data are represented as mean \pm s.d., $n=3 .{ }^{*} P<0.01$ vs untreated MCPIP1 ${ }^{-1}$ mice. The solid bar indicates MCPIP1 ${ }^{-1-}$ mice and the open bar indicates wild-type mice.

MCPIP1-deficient mice. Taken together, these results suggest that MCPIP1-deficient mice developed a lupus-like pathological changes except no glomerulonephritis.

The findings here support our original report that MCPIP1 is a negative regulator of inflammatory activation of macrophages, ${ }^{4}$ and extend our subsequent observations of the spontaneous, fatal, inflammatory disease that develops in MCPIP1-deficient mice. ${ }^{8}$ In a report from Akira's group, Zc3h12a-deficient mice generated with a mixed C57BL/6 and 129/svj background showed a phenotype similar to our mouse line, ${ }^{9}$ suggesting that MCPIP1/Zc3h12a is essential to control inflammatory response and immune homeostasis. In addition, the phenotypes developed in MCPIP1-deficient mice share many similarities with mice deficient in TTP, roquin, TANK and TIPE2 and so on, ${ }^{14-17}$ suggesting that immune homeostasis is maintained by multiple-negative regulators that could not compensate for each other. For example, the phenotypes of MCPIP1-deficient mice are resembled with the sanroque homozygous mice, including enlarged spleen and lymph nodes, anemia, hepatitis, autoantibody production and accumulation of IgG-containing immnue complexes. ${ }^{15}$ However, MCPIP1-deficenct mice did not show marked proliferative glomerolonephritis as the sanroque mice and MCPIP1-deficient mice showed obvious abnormalities in the innate arm that sanroque mice did not show. In addition, MCPIP1-deficient mice showed many phenotypes that resembled with TTP-knockout mice such as inflammatory arthritis, dermatitis, cachexia and autoimmunity. ${ }^{14}$ In TTP-knockout mice, the phenotypes can be largely attributed to the incresed production of TNF $\alpha$, whereas MCPIP1-deficient mice showed profound changes of many inflammatiory cytokines. In a recent report, myloid-specific TTP-knockout mice showed extremely sensitive to lipopolysaccharide-induced septic shock, ${ }^{18}$ which is very similar to MCPIP1-deficent mice. ${ }^{10}$ Considering roquin (sanroque), TTP (zfp36) and MCPIP1 (Zc3h12a) are all CCCH-zinc finger containing proteins, they may share some similar role and mechanisms in maintaing immune homeostasis.

Under physiological conditions, the commensal microbiota of mucosal surfaces represents the largest source of ligands for Toll-like receptors, which serve as the principle sensors of the innate immune system. It has also been reported that Toll-like receptor signaling by this commensal microbiota contributes to the pathogenesis of autoimmune diseases. ${ }^{12,13}$ To test whether commensal microbiota contributed to the pathogenesis of inflammatory disease in MCPIP1deficient mice, we treated these mice for 10 weeks with a combination of antibiotics (ampicillin, neomycin, vancomycin and metronidazole) that would have a collective broad spectrum of antibacterial activity, and would be expected to eradicate much of commensal microbiota from the intestine and other mucosal surfaces. Importantly, treatment of MCPIP1-deficient mice with antibiotics reduced abnormalities observed in the spleens, increased survival and decreased mRNA levels for inflammatory cytokines. Consequently, this series of experiments clearly supported the conclusion that commensal microbiota contributed to the development of the inflammatory and immune disorders developing in MCPIP1-deficient mice.

The molecular mechanisms underlying the pathological changes in MCPIP1-deficient mice are still not completely understood. The initial report ${ }^{3}$ that MCPIP1 may be a transcription factor is not supported by following studies. Emerging data indicated that MCPIP1 is a novel RNase, which may selectively target mRNA of 
some inflammatory cytokines such as IL-6, IL-1 $\beta$, IL-2 and IL-12p40 for degradation., ${ }^{9,-21}$ However, how MCPIP1 selectively target specific substrates is not clear. We recently observed that MCPIP1 forms granule-like structure in cytosol and is associated with the key components of RNA-induced silencing complex, such as Argnaute 2 and GW-182 (Qi et al. ${ }^{22}$ ). These data suggest that MCPIP1 may cooperate with microRNA effector pathway to silence the expression of specific inflammatory mRNAs. The previous work from us and others also suggests that MCPIP1 can inhibit inflammatory signaling especially JNK and nuclear factor- $\kappa \mathrm{B}$ signal pathways. ${ }^{8,23,24}$ Taken together, these results suggest that MCPIP1 may function as a multifunctional molecule that downregulates inflammatory and immue responses through multiple mechanisms.

\section{METHODS}

\section{Mice}

MCPIP1-deficient (MCPIP1 $1^{-/-}$) mice and their wild-type (MCPIP1 $1^{+/+}$) littermates on a C57BL/6 background were generated as described previously, ${ }^{8}$ and housed in the Laboratory Research Animal Center at the University of Missouri-Kansas City. All mice were maintained in sterilized filter-top cages and fed autoclaved food and water under specific pathogen-free conditions. Both male and female mice used were between the ages of 6 and 12 weeks, unless indicated otherwise. Experimental procedures were approved by the Animal Care and Use Committee of University of Missouri-Kansas City.

\section{Histological analyses}

Tissue samples were fixed in $10 \%$ neutral buffered formalin for 1 day, subsequently routinely processed and embedded in paraffin. Sections of $5 \mu \mathrm{m}$ thickness were stained with hematoxylin and eosin. Slides were interpreted by a board-certified pathologist blinded to the genotypes of the specimens.

\section{Immunohistochemistry and immunofluorescence}

Five micrometers sections of paraffin-embedded material were used for the immunologic staining. After rehydration, endogenous peroxidase was quenched by incubation of the sections in $0.3 \% \mathrm{H}_{2} \mathrm{O}_{2}$ diluted in methanol for $30 \mathrm{~min}$. Thereafter, antigen retrieval was performed using either citrate $(\mathrm{pH} 6.0)$ or EDTA ( $\mathrm{pH} \mathrm{8.0)}$ for $15 \mathrm{~min}$ in the autoclave. After cooling, the slides were rinsed with phosphate-buffered saline containing $0.5 \%$ Triton $\mathrm{X}-100(5 \mathrm{~min})$, followed by phosphate-buffered saline only $(3 \times 5 \mathrm{~min})$. After preincubation with normal donkey serum or normal goat serum, antibodies against CD45 and F4/80 (Abcam, Cambridge, MA, USA), DyLight 488 antimouse IgM, and DyLight 594 anti-mouse IgG (Vector Labs, Burlingame, CA, USA), were used for the analysis. CD45 (marker of B cells) and F4/80 (marker of macrophages) were detected with secondary antibody conjugated to horseradish peroxidase, followed by the addition of a chromogen substrate. DyLight 488 anti-mouse IgM and DyLight 594 anti-mouse IgG were used for immunofluorescence. Sections were counterstained with hematoxylin and $4^{\prime}, 6^{\prime}$-diamidino-2-phenylindole for immunohistochemistry and immunofluorescence, respectively.

\section{Flow cytometry}

Mouse spleen cells were collected and the red blood cells were lysed with BD lysing buffer (BD Biosciences, San Jose, CA, USA). The non-lysed cells were further stained with antibodies against surface markers to distinguish subpopulations. For cytokine expression assay, the cells were first cultured with RPMI1640 medium containing 10\% fetal bovine serum (Hyclone, South Logan, UT, USA) for $5 \mathrm{~h}$ in the presence of $1 \mu \mathrm{g} \mathrm{ml}^{-1}$ brefeldin A (BFA), $1 \mu \mathrm{g} \mathrm{ml}^{-1}$ ionomycin and $50 \mathrm{ng} \mathrm{ml}^{-1}$ phorbol myristate acetate (PMA) (all from Sigma-Aldrich, St Louis, MO, USA) and then labeled. All of the intracellular labeling was performed after the surface marker staining with Foxp3 staining buffer set (eBioscience, San Diego, CA, USA) following the manufacturer's instructions. The flow-cytometry antibodies used include anti-
CD3e (PerCP-Cy5.5), anti-B220 (phycoerythrin, PE-Cy7), anti-CD4 (allophycocyanin), anti-CD8 (fluorescein isothiocyanate isomer, FITC), anti-CD62L $(\mathrm{PE})$, anti-IFN- $\gamma(\mathrm{PE})$, anti-IL-4 (PE), anti-IL-17 (PE), anti-Foxp3 (PE), antiCD11b (PE), anti-CD115 (PerCP-eFluor710), anti-Ly-6C (APC) (all the above from eBioscience), anti-CD138 (APC) and anti-Ly-6G (FITC) (both from BD Biosciences) antibodies. Data analysis were performed using the CellQuest software (BD Bioscience).

\section{Enzyme-linked immuno sorbent assay and quantitative PCR}

Enzyme-linked immuno sorbent assay was performed by using Bio-Plex Pro (Bio-Rad, Hercules, CA, USA) Mouse Cytokine 23-plex assay according to the manufacturer's instruction. Real-time PCRs were performed using SYBR green PCR master mix (Applied Biosystems, Foster City, CA, USA) on a StepOnePlus real-time PCR system (Applied Biosystems) following standard procedures. Briefly, single-stranded complementary DNA was synthesized from $1 \mu \mathrm{g}$ of total RNA using High Capacity complementary DNA Reverse Transcription Kit (Applied Biosystems). For PCR reactions, $1 \mu \mathrm{l}$ of DNA template, $0.3 \mu \mathrm{l}$ of forward and reverse primers $\left(10 \mu \mathrm{moll}^{-1}\right.$ each $), 10 \mu \mathrm{l}$ of SYBR green PCR Master Mix and $8.4 \mu \mathrm{l}$ of water were added to a final volume of $20 \mu \mathrm{l}$. Thermal cycling was performed as follows: $95^{\circ} \mathrm{C}$ for $3 \mathrm{~min}$ as initial denaturing, followed by 40 cycles of $94^{\circ} \mathrm{C}$ for $30 \mathrm{~s}, 60^{\circ} \mathrm{C}$ for $30 \mathrm{~s}$, and a final extension at $72{ }^{\circ} \mathrm{C}$ for $2 \mathrm{~min}$. Threshold cycles $\left(\mathrm{C}_{\mathrm{T}}\right.$ values $)$ were determined using the StepOne software (Applied Biosystems). The sequence of the primers used for the PCR was shown in Supplementary Table S1. Real-time PCR results were normalized using $\beta$-actin as an internal control. Relative mRNA levels were calculated from the $C_{T}$ values for each sample.

\section{Antibiotic treatment}

Both MCPIP1-deficient mice and their wild-type littermates received untreated water or treated water containing ampicillin $\left(1 \mathrm{gl}^{-1}\right)$, neomycin $\left(1 \mathrm{gl}^{-1}\right)$, vancomycin $\left(0.5 \mathrm{gl}^{-1}\right)$ and metronidazole $\left(1 \mathrm{gl}^{-1}\right)$ from birth to 10 weeks. The body weight and survival rate were recorded. At 10 weeks, surviving mice were killed and the spleens were weighed and photographed. Cytokine expression in the spleen was measured by quantitative PCR.

\section{Statistics}

Data were expressed as mean \pm s.d. For comparison between two groups the unpaired Student's test was used. For multiple comparisons, analysis of variance followed by unpaired Student's test was used. A value of $P<0.05$ was considered significant.

\section{CONFLICT OF INTEREST}

The authors declare no conflict of interest.

\section{ACKNOWLEDGEMENTS}

We thank Ms. Ruth E Morgan for helping with the experiments of flow cytometry. Flow cytometry was performed in the Children's Mercy Hospital and Clinics Core Clinical and Research Flow Cytometry Laboratory. This work was partially supported by the National Institutes of Health grants (HL098794 to MF, HL097218 and HL076206 to GL, HL068878 and HL089544 to YEC). YEC is an Established Investigator of the American Heart Association $(0840025 \mathrm{~N})$

1 Van Parijs L, Abbas AK. Homeostasis and self-tolerance in the immune system: turning lymphocytes off. Science 1998; 280: 243-248.

2 Stockinger B, Kassiotis G, Bourgeois C. Homeostasis and T cell regulation. Curr Opin Immunol 2004; 16: 775-779.

3 Zhou L, Azfer A, Niu J, Graham S, Choudhury M, Adamski FM et al. Monocyte chemoattractant protein-1 induces a novel transcription factor that causes cardiac myocyte apoptosis and ventricular dysfunction. Circ Res 2006; 98: 1177-1185.

4 Liang J, Wang J, Azfer A, Song W, Tromp G, Kolattukudy PE et al. A novel CCCH-zinc finger protein family regulates proinflammatory activation of macrophages. $\mathrm{J} \mathrm{Biol} \mathrm{Chem}$ 2008; 283: 6337-6346.

5 Liang J, Song W, Tromp G, Kolattukudy PE, Fu M. Genome-wide survey and expression profiling of $\mathrm{CCCH}$-zinc finger family reveals a functional module in macrophage activation. PLoS One 2008; 3: e2880. 
6 Skalniak L, Mizgalska D, Zarebski A, Wyrzykowska P, Koj A, Jura J. Regulatory feedback loop between NF-kappaB and MCP-1-induced protein 1 RNase. FEBS $J$ 2009; 276: 5892-5905.

7 Kasza A, Wyrzykowska P, Horwacik I, Tymoszuk P, Mizgalska D, Palmer $\mathrm{K}$ et al. Transcription factors Elk-1 and SRF are engaged in IL1-dependent regulation of ZC3H12A expression. BMC Mol Biol 2010; 11: 14

8 Liang J, Saad Y, Lei T, Wang J, Qi D, Yang $Q$ et al. MCP-induced protein 1 deubiquitinates TRAF proteins and negatively regulates JNK and NF-kappaB signaling. J Exp Med 2010; 207: 2959-2973.

9 Matsushita K, Takeuchi O, Standley DM, Kumagai Y, Kawagoe T, Miyake T et al. Zc3h12a is an RNase essential for controlling immune responses by regulating mRNA decay. Nature 2009; 458: 1185-1190.

10 Huang S, Miao R, Zhou Z, Wang T, Liu J, Liu G et al. MCPIP1 negatively regulates Tolllike receptor 4 signaling and protects mice from LPS-induced septic shock. Cell Signal 2013; 25: 1228-1234.

11 Lin RJ, Chien HL, Lin SY, Chang BL, Yu HP, Tang WC et al. MCPIP1 ribonuclease exhibits broad-spectrum antiviral effects through viral RNA binding and degradation. Nucleic Acids Res 2013; 41: 3314-3326.

12 Saleh M, Elson CO. Experimental inflammatory bowel disease: insights into the hostmicrobiota dialog. Immunity 2011; 34: 293-302.

13 Feng T, Elson CO. Adaptive immunity in the host-microbiota dialog. Mucosal Immunol $2011 ; 4: 15-21$.

14 Taylor GA, Carballo E, Lee DM, Lai WS, Thompson MJ, Patel DD et al. A pathogenetic role for TNFalpha in the syndrome of cachexia, arthritis, and autoimmunity resulting from tristetraprolin (TTP) deficiency. Immunity 1996; 4: 445-454.

15 Vinuesa CG, Cook MC, Angelucci C, Athanasopoulos V, Rui L, Hill KM et al. A RINGtype ubiquitin ligase family member required to repress follicular helper $\mathrm{T}$ cells and autoimmunity. Nature 2005; 435: 452-458.
16 Kawagoe T, Takeuchi O, Takabatake Y, Kato H, Isaka Y, Tsujimura T et al. TANK is a negative regulator of Toll-like receptor signaling and is critical for the prevention of autoimmune nephritis. Nat Immunol 2009; 10: 965-972.

17 Sun H, Gong S, Carmody RJ, Hilliard A, Li L, Sun J et al. TIPE2, a negative regulator of innate and adaptive immunity that maintains immune homeostasis. Cell 2008; 133 415-426.

18 Qiu LQ, Stumpo DJ, Blackshear PJ. Myeloid-specific tristetraprolin deficiency in mice results in extreme lipopolysaccharide sensitivity in an otherwise minimal phenotype. J Immunol 2012; 188: 5150-5159.

19 Mizgalska D, Wegrzyn P, Murzyn K, Kasza A, Koj A, Jura J et al. Interleukin-1-inducible MCPIP protein has structural and functional properties of RNase and participates in degradation of IL-1beta mRNA. FEBS J 2009; 276: 7386-7399.

20 Xu J, Peng W, Sun Y, Wang X, Xu Y, Li X et al. Structural study of MCPIP1 N-terminal conserved domain reveals a PIN-like RNase. Nucleic Acids Res 2012; 40: 6957-6965.

$21 \mathrm{Li} \mathrm{M,} \mathrm{Cao} \mathrm{W,} \mathrm{Liu} \mathrm{H,} \mathrm{Zhang} \mathrm{W,} \mathrm{Liu} \mathrm{X,} \mathrm{Cai} \mathrm{Z} \mathrm{et} \mathrm{al.} \mathrm{MCPIP1} \mathrm{down-regulates}$ IL-2 expression through an ARE-independent pathway. PLoS One 2012; 7: e49841.

22 Qi D, Huang S, Miao R, She ZG, Quinn T, Chang Y et al. Monocyte chemotactic proteininduced protein 1 (MCPIP1) suppresses stress granule formation and determines apoptosis under stress. J Biol Chem 2011; 286: 41692-41700.

23 Liang J, Wang J, Saad Y, Warble L, Becerra E, Kolattukudy PE. Participation of MCP-induced protein 1 in lipopolysaccharide preconditioning-induced ischemic stroke tolerance by regulating the expression of proinflammatory cytokines. J Neuroinflammation 2011; 8: 182.

24 Niu J, Wang K, Graham S, Azfer A, Kolattukudy PE. MCP-1-induced protein attenuates endotoxin-induced myocardial dysfunction by suppressing cardiac NF-kB activation via inhibition of $I_{\kappa} B$ kinase activation. J Mol Cell Cardiol 2011; 51: 177-186.

The Supplementary Information that accompanies this paper is available on the Immunology and Cell Biology website (http://www.nature.com/icb) 